\title{
Hyperglycemia and Adverse Pregnancy Outcome Follow-Up Study: newborn anthropometrics and childhood glucose metabolism
}

\author{
Monica E. Bianco ${ }^{1,2}$ (D) - Alan Kuang ${ }^{3}$ (D) - Jami L. Josefson ${ }^{1,2}$ (D) Patrick M. Catalano ${ }^{4}$ (D) Alan R. Dyer ${ }^{3} \cdot$ Lynn P. Lowe $^{3}$. \\ Boyd E. Metzger ${ }^{5}$ (D) - Denise M. Scholtens ${ }^{3}$ (D) - William L. Lowe $\mathrm{Jr}^{5}$ (D) - on behalf of the HAPO Follow-Up Study \\ Cooperative Research Group
}

Received: 29 June 2020 / Accepted: 2 October 2020

(C) Springer-Verlag GmbH Germany, part of Springer Nature 2020

\begin{abstract}
Aims/hypothesis We aimed to examine associations of newborn anthropometric measures with childhood glucose metabolism with the hypothesis that greater newborn birthweight, adiposity and cord C-peptide are associated with higher childhood glucose levels and lower insulin sensitivity.

Methods Data from the international, multi-ethnic, population-based Hyperglycemia and Adverse Pregnancy Outcome (HAPO) Study and the HAPO Follow-Up Study were used. The analytic cohort included 4155 children (mean age [SD], 11.4 [1.2] years; $51.0 \%$ male). Multiple linear regression was used to examine associations of primary predictors, birthweight, newborn sum of skinfolds (SSF) and cord C-peptide, from HAPO with continuous child glucose outcomes from the HAPO Follow-Up Study. Results In an initial model that included family history of diabetes and maternal BMI during pregnancy, birthweight and SSF demonstrated a significant, inverse association with $30 \mathrm{~min}$ and $1 \mathrm{~h}$ plasma glucose levels. In the primary model, which included further adjustment for maternal sum of glucose $\mathrm{z}$ scores from an oral glucose tolerance test during pregnancy, the associations were strengthened, and birthweight and SSF were inversely associated with fasting, $30 \mathrm{~min}, 1 \mathrm{~h}$ and $2 \mathrm{~h}$ plasma glucose levels. Birthweight and SSF were also associated with higher insulin sensitivity (Matsuda index) $(\beta=1.388$; 95\% CI 0.870, 1.906; $p<0.001 ; \beta=0.792 ; 95 \%$ CI $0.340,1.244 ; p<0.001$, for birthweight and SSF higher by $1 \mathrm{SD}$, respectively) in the primary model, while SSF, but not birthweight, was positively associated with the disposition index, a measure of beta cell compensation for insulin resistance $(\beta=0.034 ; 95 \%$ CI $0.012,0.056 ; p=0.002)$. Cord C-peptide levels were inversely associated with Matsuda index ( $\beta=-0.746 ; 95 \% \mathrm{CI}-1.188,-0.304 ; p<0.001$ for cord C-peptide higher by $1 \mathrm{SD}$ ) in the primary model.

Conclusions/interpretation This study demonstrates that higher birthweight and SSF are associated with greater childhood insulin sensitivity and lower glucose levels following a glucose load, associations that were further strengthened after adjustment for maternal glucose levels during pregnancy.
\end{abstract}

William L. Lowe, Jr.

wlowe@northwestern.edu

1 Ann \& Robert H. Lurie Children's Hospital of Chicago, Chicago, IL, USA

2 Department of Pediatrics, Northwestern University Feinberg School of Medicine, Chicago, IL, USA

3 Department of Preventive Medicine, Northwestern University Feinberg School of Medicine, Chicago, IL, USA

4 Mother Infant Research Institute, Tufts University School of Medicine, Boston, MA, USA

5 Department of Medicine, Northwestern University Feinberg School of Medicine, Chicago, IL, USA 


\section{Research in context}

\section{What is already known about this subject?}

- Associations of birthweight with adult metabolic diseases have been studied

- Few studies have examined the association of birthweight and adiposity with measures of childhood plasma glucose

What is the key question?

- What is the relationship of newborn birthweight, adiposity and cord C-peptide levels to childhood glucose levels and insulin sensitivity?

What are the new findings?

- Higher birthweight and newborn adiposity, as measured by sum of skinfolds, were associated with lower glucose levels and greater insulin sensitivity in childhood

- The above relationships were strengthened after adjustment for maternal glucose levels during pregnancy

- Cord C-peptide levels were inversely associated with insulin sensitivity in childhood

\section{How might this impact on clinical practice in the foreseeable future?}

- The findings could lead to a reconsideration of the implications of size at birth, as greater size at birth is associated with improved metabolic outcomes during early childhood; however, it is possible that excess adiposity that accumulates in utero secondary to maternal plasma glucose levels and/or obesity may contribute to adverse metabolic outcomes

Keywords Birthweight - Childhood glucose metabolism · Childhood plasma glucose $\cdot$ Fetal exposures · Newborn adiposity Newborn anthropometrics

\author{
Abbreviations \\ FPG Fasting plasma glucose \\ FUS Follow-Up Study \\ HAPO Hyperglycemia and Adverse Pregnancy Outcome \\ oDI Oral disposition index \\ PG Plasma glucose \\ SGA Small for gestational age \\ SSF Sum of skinfolds
}

\section{Introduction}

The prevalence of youth-onset type 2 diabetes in the USA rose by $35 \%$ between 2001 and 2009, with an expectation that its prevalence will quadruple by $2050[1,2]$. Youth-onset type 2 diabetes is associated with high therapeutic failure rates and rapid progression to diabetes-related complications, suggesting a more aggressive disease process in children [3-5]. Similarly, the prevalence of childhood disorders of glucose metabolism appears to be increasing together with the increasing prevalence of childhood obesity [6]. As childhood disorders of glucose metabolism are a precursor to diabetes, identifying how and when to intervene with preventive strategies is an area of interest.

Maternal plasma glucose (PG) levels are associated with newborn anthropometrics as well as childhood obesity and disorders of glucose metabolism [7, 8], but the association of newborn anthropometrics with childhood glucose metabolism is less clear [9-13]. Nevertheless, the known associations of maternal hyperglycaemia have led to efforts to prevent large for gestational age newborns as a potential indicator of future metabolic disease. However, size at birth is determined by both fetal genetic factors and the intrauterine environment, as determined by maternal PG, obesity and other factors [8, 14]. Associations of birthweight with adult metabolic disease have been examined, although these studies often did not account for maternal PG and/or obesity during pregnancy in the analyses [15-17]. Previous studies examining the association of newborn size at birth with childhood metabolic outcomes have had limitations, including restriction to children of European ancestry, reliance on maternal recall of birthweight and/or no consideration of maternal metabolic variables during pregnancy [9-13]. Thus, the association of newborn anthropometrics with childhood metabolic health as a potential harbinger of future metabolic disease is incompletely understood. 
To address this, we analysed maternal, newborn and childhood data from the Hyperglycemia and Adverse Pregnancy Outcome (HAPO) Study and the HAPO Follow-Up Study (FUS), which included a large, population-based, international cohort of mother-offspring pairs for whom maternal metabolic data at $\sim 28$ weeks of gestation and offspring data at birth and age 10-14 years were available. HAPO demonstrated positive and additive associations of maternal PG and BMI during pregnancy with newborn size at birth and cord Cpeptide $[8,18]$, while the HAPO FUS demonstrated that maternal hyperglycaemia during pregnancy was associated with higher glucose levels, lower insulin sensitivity and a higher frequency of disorders of glucose metabolism during childhood [19, 20]. Given these previously reported associations, the present study examined the hypothesis that greater newborn birthweight, adiposity and cord C-peptide are associated with higher childhood glucose levels and lower insulin sensitivity.

\section{Methods}

HAPO study HAPO was a prospective, population-based study in which women underwent a $75 \mathrm{~g}$ OGTT at $\sim 28$ weeks of gestation [8]. OGTT results remained blinded to caregivers and participants except for fasting plasma glucose (FPG) $>105 \mathrm{mg} / \mathrm{dl}(5.8 \mathrm{mmol} / \mathrm{l})$ and/or $2 \mathrm{~h} \mathrm{PG}>200 \mathrm{mg} / \mathrm{dl}$ $(11.1 \mathrm{mmol} / \mathrm{l})$, either measure $<45 \mathrm{mg} / \mathrm{dl}(2.5 \mathrm{mmol} / \mathrm{l})$ or random $P G$ at $34-37$ weeks gestation $\geq 160 \mathrm{mg} / \mathrm{dl}$ $(8.9 \mathrm{mmol} / \mathrm{l})$ [8]. Blinded participants were untreated. The sum of individual glucose $\mathrm{z}$ scores at fasting, $1 \mathrm{~h}$ and $2 \mathrm{~h}$ during the pregnancy OGTT, an integrated measure that gives equal weight to each of the three glucose values, was used in statistical analyses. The $\mathrm{z}$ scores were calculated by first subtracting the sample mean and then dividing by the sample SD for each timed glucose measurement from the OGTT, using sample means and SDs from the full HAPO cohort. Observed distributions for each timed glucose measurement were approximately normal. Individual z scores were then summed for use as a composite measure in statistical modelling. Height, weight and blood pressure were measured at the OGTT using standardised procedures. Demographic and lifestyle characteristics, including age, self-reported race and ethnicity, smoking or alcohol use during pregnancy, and marital and employment status were collected via questionnaire and parity by medical record abstraction. Cord blood samples were obtained within $5 \mathrm{~min}$ of delivery and before delivery of the placenta. Cord blood C-peptide, birthweight and sum of skinfolds (SSF) were measured as described [8] using calibrated equipment and standardised methods [18].

HAPO FUS participants HAPO FUS participants were recruited between 2013 and 2016 from ten of 15 HAPO field centres based on recruitment feasibility [21]. HAPO FUS eligibility criteria included caregivers and participants being blinded to the HAPO OGTT results, gestational age at delivery $\geq 37$ weeks and no major neonatal malformations or fetal/ neonatal death. This yielded 15,812 eligible mother-child pairs. The recruitment target was 7000 pairs, based on the primary childhood outcome of overweight/obesity [21]. A total of 4834 children completed all or part of the HAPO FUS visit; 4155 children did not have a pre-existing diagnosis of diabetes and had a fasting glucose measurement and at least one other timed OGTT measurement. The cohort of 4155 children included five children who had glucose levels during the OGTT consistent with a diagnosis of diabetes and were negative for serum autoantibodies (see below).

The study protocol was approved by each centre's institutional review board. Mothers gave written, informed consent for their child; children assented where required by the local institutional review board. There was an external Observational Study Monitoring Board.

HAPO FUS visit Child height and weight were measured, and a $2 \mathrm{~h}$ OGTT with a glucose load of $1.75 \mathrm{~g} / \mathrm{kg}$ body weight (maximum $75 \mathrm{~g}$ ) following an $8 \mathrm{~h}$ overnight fast with samples drawn for glucose and C-peptide at fasting, $30 \mathrm{~min}, 1 \mathrm{~h}$ and $2 \mathrm{~h}$ was performed as described previously [19-21]. Samples were processed at the field centre laboratory and stored at $-80^{\circ} \mathrm{C}$ until shipment to the Central Laboratory.

The child's age and family history of diabetes in firstdegree relatives were collected from the mother via questionnaire.

Laboratory measurements Glucose was measured by hexokinase in the Northwestern Memorial Hospital Clinical Chemistry Laboratory on a Beckman-Coulter SYNCHRON LX analyser (Beckman Coulter, Chaska, MN, USA) with blinded duplicate samples assayed for a random subset of $10 \%$ as described $[19,20]$. C-peptide was measured in the Northwestern Comprehensive Metabolic Core by electrochemiluminescence immunoassay on a Roche cobas e411 immunoassay analyser (Roche Diagnostics, Indianapolis, IN, USA) $[19,20]$. Type 1 diabetes was diagnosed by measuring serum autoantibodies $[19,20]$ for children reported to have diabetes on treatment $(n=9)$ or OGTT values indicative of incident diabetes $(n=5)$. All children with diabetes on treatment or type 1 diabetes were excluded from the analyses.

Outcomes and predictors The primary predictors for childhood outcomes were birthweight, newborn SSF and cord blood C-peptide, which were measured as described [8].

Continuous childhood outcomes included FPG and $30 \mathrm{~min}$, $1 \mathrm{~h}$ and $2 \mathrm{~h} \mathrm{PG}$ from the OGTT. Similar to the maternal pregnancy OGTT values, the sum of individual glucose $\mathrm{z}$ 
scores, i.e., the four time points for the child's OGTT from the HAPO FUS, was also examined [19]. Observed distributions for each timed glucose measurement in children were approximately normal.

Matsuda, insulinogenic and disposition indices were also examined as continuous outcomes. A modified Matsuda index, which estimates insulin sensitivity, was calculated using OGTT glucose and C-peptide levels [22]. The insulinogenic index, a measure of insulin secretion, was calculated as described [23]. The oral disposition index (oDI), a measure of beta cell compensation for insulin resistance, was calculated as the product of the Matsuda and insulinogenic indices and then log transformed [24].

Statistical analyses Data were summarised using frequencies and counts for categorical variables and means and SDs for continuous variables. Histograms and box plots were examined to assess distributions and identify potential outliers. Quantile regression was used to generate categorical quantiles of SSF, adjusted for field centre and gestational age at delivery; quantile estimates were made separately according to newborn sex. Multiple linear regression was used to estimate adjusted mean differences with $95 \%$ CIs for continuous outcomes. Quadratic terms and restricted cubic splines estimated with the rms R package version 5.1-2 (https://cran.rproject.org/src/contrib/Archive/rms/rms_5.1-2.tar.gz, accessed 30 March 2018) were used to assess linearity [25].

Two models were considered, with variables identified according to study design, known potential confounders and adjustments used in HAPO analyses [8]. The primary predictors for each of the models were birthweight, newborn SSF and cord C-peptide, while the covariate adjustments for the different models were as follows: model 1: field centre (proxy for race/ancestry since most centres were predominantly one race/ancestry group), child age and sex, maternal variables at HAPO pregnancy OGTT (age, height, mean arterial pressure, parity [0 or $1+]$, smoking [yes/no], drinking alcohol [yes/no] and gestational age) and child's family history of diabetes in first-degree relatives + maternal BMI at HAPO pregnancy OGTT; model 2: model $1+$ maternal sum of glucose $\mathrm{z}$ scores from the HAPO pregnancy OGTT. Adjustments for maternal glucose sum of $\mathrm{z}$ scores and BMI were included in the primary model as each was independently associated with birthweight and newborn SSF in the HAPO cohort $[8,26]$. Multiple imputation was performed for the full analytic dataset including predictors, outcomes and covariates using chained equations as implemented in the mice $\mathrm{R}$ package version 2.30 (https:// cran.r-project.org/src/contrib/Archive/mice/mice_2.30.tar.gz, accessed 12 October 2018) and 25 imputed datasets [27]. Imputations were performed under a 'missing at random' assumption after confirming findings varied little under 'missing not at random' [28]. Two-sided $p<0.05$ was used for evaluating statistical significance. All statistical analyses
Table 1 Characteristics of mothers during HAPO pregnancy and children at follow-up

\begin{tabular}{|c|c|}
\hline Characteristic & Overall $n=4155$ \\
\hline \multicolumn{2}{|c|}{ Characteristics of mothers during HAPO pregnancy } \\
\hline Age (years) & $29.89 \pm 5.68$ \\
\hline Height $(\mathrm{cm})$ & $161.65 \pm 6.79$ \\
\hline Weight at OGTT $(\mathrm{kg})$ & $71.77 \pm 14.14$ \\
\hline BMI & $27.43 \pm 4.91$ \\
\hline Gestational age at OGTT (weeks) & $27.60 \pm 1.70$ \\
\hline Mean arterial pressure $(\mathrm{mmHg})$ & $80.25 \pm 7.88$ \\
\hline FPG level $(\mathrm{mmol} / \mathrm{l})$ & $4.49 \pm 0.37$ \\
\hline $1 \mathrm{~h} \mathrm{PG}$ level $(\mathrm{mmol} / \mathrm{l})$ & $7.40 \pm 1.68$ \\
\hline 2 h PG level (mmol/l) & $6.15 \pm 1.29$ \\
\hline Sum of PG z scores & $-0.06 \pm 2.31$ \\
\hline \multicolumn{2}{|l|}{ Ancestry group } \\
\hline Asian & $1125(27.1)$ \\
\hline Black & $719(17.3)$ \\
\hline Hispanic & $465(11.2)$ \\
\hline White & $1773(42.7)$ \\
\hline Other & $73(1.8)$ \\
\hline \multicolumn{2}{|l|}{ Smoking during pregnancy } \\
\hline No & $3978(95.7)$ \\
\hline Yes & $177(4.3)$ \\
\hline \multicolumn{2}{|l|}{ Alcohol intake during pregnancy } \\
\hline No & $3872(93.2)$ \\
\hline Yes & $283(6.8)$ \\
\hline \multicolumn{2}{|l|}{ Parity (any prior delivery $\geq 20$ weeks) } \\
\hline No & $2010(48.4)$ \\
\hline Yes & $2145(51.6)$ \\
\hline \multicolumn{2}{|l|}{ Child characteristics at birth } \\
\hline Gestational age at delivery (weeks) & $39.78 \pm 1.19$ \\
\hline Birthweight $(\mathrm{g})$ & $3371.39 \pm 469.19$ \\
\hline $\mathrm{SSF}(\mathrm{mm})$ & $12.31 \pm 2.55$ \\
\hline Cord C-peptide (nmol/1) & $0.33 \pm 0.19$ \\
\hline \multicolumn{2}{|l|}{ Child characteristics at follow-up } \\
\hline Age (years) & $11.36 \pm 1.22$ \\
\hline Height $(\mathrm{cm})$ & $148.52 \pm 10.36$ \\
\hline Weight $(\mathrm{kg})$ & $43.18 \pm 13.57$ \\
\hline \multicolumn{2}{|l|}{ Sex } \\
\hline Female & $2034(49.0)$ \\
\hline Male & $2121(51.0)$ \\
\hline \multicolumn{2}{|l|}{ Family history of diabetes } \\
\hline No & $2255(54.3)$ \\
\hline Yes & $1896(45.7)$ \\
\hline \multicolumn{2}{|l|}{ Child outcomes at follow-up } \\
\hline FPG level (mmol/l) & $5.00 \pm 0.39$ \\
\hline $30 \mathrm{~min}$ PG level $(\mathrm{mmol} / \mathrm{l})$ & $7.80 \pm 1.33$ \\
\hline $1 \mathrm{~h} \mathrm{PG}$ level $(\mathrm{mmol} / \mathrm{l})$ & $6.83 \pm 1.68$ \\
\hline 2 h PG level $(\mathrm{mmol} / \mathrm{l})$ & $5.97 \pm 1.15$ \\
\hline Sum of glucose z scores & $-0.01 \pm 2.83$ \\
\hline Matsuda index & $33.15 \pm 13.70$ \\
\hline Insulinogenic index & $0.14 \pm 0.12$ \\
\hline oDI & $4.21 \pm 3.97$ \\
\hline
\end{tabular}

Data are displayed as mean $\pm \mathrm{SD}$ or $n(\%)$

were conducted in R (3.3.1) (https://cran.r-project.org/bin/ windows/base/old/3.3.1/, accessed 27 July 2018) [29].

\section{Results}

Participants Characteristics of the 4155 children with OGTT results from the HAPO FUS and their mothers during the 
HAPO Study are displayed in Table 1. Mothers of children who did and did not participate (unable to contact or declined) in the HAPO FUS were similar and have been described [21] (electronic supplementary material [ESM] Table 1).

Association of newborn measures with childhood glucose levels Initial analyses examined associations of birthweight, newborn SSF and cord C-peptide with child glucose levels during the HAPO FUS OGTT (Table 2). Given previous reports from HAPO demonstrating the independent association of maternal PG and BMI with both newborn and childhood anthropometric and metabolic outcomes [8, 18-20], the primary model for the present study (model 2) included adjustment for both maternal BMI and sum of glucose $\mathrm{z}$ scores from the HAPO OGTT. To better define the relationships among maternal PG during pregnancy, newborn anthropometrics and child glucose-related outcomes, an initial model (model 1) that included adjustment for maternal BMI but not maternal glucose levels was also considered.

No newborn measures were associated with child FPG in the initial model (model 1); however, after adjustment for maternal sum of glucose z scores (model 2), birthweight and SSF were significantly and inversely associated with childhood FPG $(\beta=-0.015 ; 95 \%$ CI $-0.029,-0.001 ; p=0.041$ and $\beta=-0.019,95 \% \mathrm{CI}-0.031,-0.006 ; p=0.003$ for birthweight and SSF higher by $1 \mathrm{SD}$, respectively) (Table 2). Cord C-peptide was not associated with child FPG.

Birthweight and SSF were also inversely associated with child $30 \mathrm{~min}, 1 \mathrm{~h}$ and $2 \mathrm{~h}$ glucose levels, and sum of glucose $\mathrm{z}$ scores (Table 2). Significant inverse associations of birthweight and SSF with these four child glucose outcomes were observed in both model 1 and model 2 (except SSF was not associated with $2 \mathrm{~h}$ glucose in model 1). For each of these outcomes, associations were strengthened after adjustment for maternal glucose sum of $\mathrm{z}$ scores during pregnancy (model 2) (30 min PG: $\beta=-0.103$; 95\% CI $-0.157,-0.050$; $p<0.001$ and $\beta=-0.096 ; 95 \%$ CI $-0.142,-0.051$; $p<0.001$ for birthweight and $\mathrm{SSF}$, respectively; $1 \mathrm{~h}$ PG: $\beta=-0.128 ; 95 \%$ CI $-0.197,-0.059 ; p<0.001$ and $\beta=-0.128 ; 95 \% \mathrm{CI}-0.187,-0.069 ; p<0.001$ for birthweight and SSF, respectively; $2 \mathrm{~h}$ PG: $\beta=-0.069$; $95 \% \mathrm{CI}-0.116,-0.022 ; p=0.004$ and $\beta=-0.053 ; 95 \%$ CI $-0.093,-0.013 ; p=0.010$ for birthweight and SSF, respectively; sum of glucose $\mathrm{z}$ scores: $\beta=-0.252 ; 95 \%$ CI $-0.361,-0.143 ; p<0.001$ and $\beta=-0.242 ; 95 \% \mathrm{CI}$ $-0.335,-0.148 ; p<0.001$ for birthweight and SSF, respectively; all $\beta$ estimates are for birthweight or SSF higher by $1 \mathrm{SD})$. Further adjustment for maternal marital and employment status at the time of the HAPO OGTT as a proxy for maternal socioeconomic status did not change these associations (ESM Table 2). Cord C-peptide was not associated with child $30 \mathrm{~min}$,
$1 \mathrm{~h}$ or $2 \mathrm{~h}$ glucose levels or sum of glucose $\mathrm{z}$ scores (Table 2).

Association of newborn measures with childhood insulin sensitivity and secretion Neither birthweight, nor SSF, nor cord C-peptide was significantly associated with childhood insulinogenic index, a measure of insulin secretion, in either model 1 or model 2 (ESM Table 3).

In contrast, birthweight, SSF and cord C-peptide demonstrated significant associations with the Matsuda index (i.e., child insulin sensitivity) (Fig. 1). Birthweight was positively associated with insulin sensitivity (i.e., children with higher birthweight were more insulin sensitive in childhood) in model 1 , and the strength of this association increased slightly after adjusting for maternal sum of glucose $\mathrm{z}$ scores (model 2, $\beta=1.388 ; 95 \%$ CI $0.870,1.906 ; p<0.001$ for birthweight higher by $1 \mathrm{SD})$. SSF was also positively associated with childhood insulin sensitivity in model 1 with a modest strengthening of the association after adjustment for maternal sum of glucose z scores (model 2, $\beta=0.792 ; 95 \%$ CI 0.340 , 1.244; $p<0.001$ for SSF higher by $1 \mathrm{SD}$ ). In contrast to the above positive associations, cord C-peptide was inversely associated with child insulin sensitivity in models 1 and 2 . The strength of this association was attenuated slightly by adjusting for maternal sum of glucose $\mathrm{z}$ scores (model 2, $\beta=-0.746 ; 95 \%$ CI $-1.188,-0.304 ; p<0.001$ for cord Cpeptide higher by $1 \mathrm{SD}$ ).

Finally, associations of birthweight, SSF and cord Cpeptide with the oDI during childhood were examined. Neither birthweight nor cord C-peptide was associated with child oDI (Fig. 1). A positive association of SSF with the oDI was observed in model 2 which included adjustment for both maternal BMI and sum of glucose $\mathrm{z}$ scores during the pregnancy OGTT (model 2, $\beta=0.034 ; 95 \%$ CI 0.012, 0.056; $p=$ 0.002 for SSF higher by $1 \mathrm{SD})$. Once again, further adjustment for maternal employment and marital status at the time of the HAPO Study OGTT did not change associations of birthweight, SSF and cord C-peptide with either child insulin sensitivity or oDI (ESM Table 2).

Measures of child glucose metabolism across quantiles of SSF Being small for gestational age (SGA) due to growth restriction and macrosomia is associated with type 2 diabetes in adults [30, 31]. As newborns with the former typically have less body fat than constitutionally SGA newborns while newborns with macrosomia typically have more body fat than constitutionally large newborns, child glucose levels during the OGTT, Matsuda index and oDI across quantiles of newborn SSF were examined (Fig. 2). A pattern of declining glucose levels and increasing oDI across quantiles of newborn SSF was observed. For insulin sensitivity, similar levels were present in the highest and lowest quantiles with some suggestion of nadir and peak levels in the middle quantiles. However, 
Table 2 Associations of newborn measures with childhood glucose levels

\begin{tabular}{|c|c|c|c|c|c|c|c|c|c|}
\hline \multirow[t]{2}{*}{ Variable } & \multicolumn{3}{|c|}{ Birthweight } & \multicolumn{3}{|l|}{ SSF } & \multicolumn{3}{|c|}{ Cord C-peptide } \\
\hline & $\beta^{a}$ & $95 \% \mathrm{CI}$ & $p$ value & $\beta^{a}$ & $95 \% \mathrm{CI}$ & $p$ value & $\beta^{a}$ & $95 \% \mathrm{CI}$ & $p$ value \\
\hline \multicolumn{10}{|c|}{ Fasting glucose $(\mathrm{mmol} / \mathrm{l})$} \\
\hline Model 1 & -0.008 & $-0.022,0.006$ & 0.271 & -0.012 & $-0.024,0.000$ & 0.057 & -0.004 & $-0.016,0.008$ & 0.535 \\
\hline Model 2 & -0.015 & $-0.029,-0.001$ & 0.041 & -0.019 & $-0.031,-0.006$ & 0.003 & -0.011 & $-0.023,0.001$ & 0.067 \\
\hline \multicolumn{10}{|c|}{30 min glucose $(\mathrm{mmol} / \mathrm{l})$} \\
\hline Model 1 & -0.074 & $-0.127,-0.020$ & 0.007 & -0.067 & $-0.112,-0.022$ & 0.004 & 0.001 & $-0.046,0.047$ & 0.977 \\
\hline Model 2 & -0.103 & $-0.157,-0.050$ & $<0.001$ & -0.096 & $-0.142,-0.051$ & $<0.001$ & -0.030 & $-0.078,0.017$ & 0.213 \\
\hline \multicolumn{10}{|c|}{$1 \mathrm{~h}$ glucose $(\mathrm{mmol} / \mathrm{l})$} \\
\hline Model 1 & -0.094 & $-0.162,-0.025$ & 0.007 & -0.094 & $-0.152,-0.035$ & 0.002 & -0.005 & $-0.064,0.053$ & 0.858 \\
\hline Model 2 & -0.128 & $-0.197,-0.059$ & $<0.001$ & -0.128 & $-0.187,-0.069$ & $<0.001$ & -0.041 & $-0.100,0.019$ & 0.180 \\
\hline \multicolumn{10}{|c|}{2 h glucose $(\mathrm{mmol} / \mathrm{l})$} \\
\hline Model 1 & -0.051 & $-0.098,-0.005$ & 0.031 & -0.036 & $-0.075,0.004$ & 0.080 & 0.003 & $-0.036,0.042$ & 0.887 \\
\hline Model 2 & -0.069 & $-0.116,-0.022$ & 0.004 & -0.053 & $-0.093,-0.013$ & 0.01 & -0.016 & $-0.055,0.024$ & 0.441 \\
\hline \multicolumn{10}{|c|}{ Sum of glucose z scores } \\
\hline Model 1 & -0.176 & $-0.284,-0.067$ & 0.002 & -0.166 & $-0.259,-0.073$ & $<0.001$ & -0.011 & $-0.103,0.081$ & 0.816 \\
\hline Model 2 & -0.252 & $-0.361,-0.143$ & $<0.001$ & -0.242 & $-0.335,-0.148$ & $<0.001$ & -0.091 & $-0.184,0.003$ & 0.058 \\
\hline
\end{tabular}

Model 1 was adjusted for field centre, child age and sex, maternal variables at pregnancy OGTT (age, height, mean arterial pressure, parity [0 or 1+], smoking [yes/no], drinking alcohol [yes/no] and gestational age) and child's family history of diabetes in first-degree relatives + maternal BMI at pregnancy OGTT. Model 2 was model $1+$ maternal sum of glucose z scores at pregnancy OGTT

${ }^{a} \beta$ values for child continuous outcomes are reported for newborn birthweight, SSF and cord C-peptide higher by 1 SD

as was the case for all models examined, assessments of quadratic terms and restricted cubic splines for each of the continuous predictors did not indicate a statistically significant departure from linearity for any of the outcomes.

\section{Discussion}

The present study demonstrated that higher birthweight and newborn adiposity, as measured by SSF, were associated with lower glucose levels and greater insulin sensitivity in childhood. These associations were strengthened after adjusting for a measure of maternal glucose during pregnancy, sum of glucose $\mathrm{z}$ scores during an OGTT. These findings stand in contrast to the hypothesis being addressed and suggest a complex interaction among maternal PG during pregnancy, size at birth and childhood metabolic outcomes.

Some, but not all, previous studies examining the association between size at birth and insulin sensitivity during childhood demonstrated a positive association between newborn size at birth and insulin sensitivity during childhood [9-13]. The present study expands upon these previous studies in several ways. First, earlier studies were limited to children of European ancestry, whereas the present study demonstrates the same relationship in a multi-ethnic cohort. Second, in addition to examining the association of birthweight with childhood metabolic outcomes, the present study also examined association of newborn adiposity, as reflected by SSF, with childhood metabolic outcomes. Third, the present study adjusted for maternal BMI and PG during pregnancy; adjustment for the latter, an important contributor to fetal fat accretion, was a unique aspect of this study. Finally, associations of size at birth with multiple childhood metabolic outcomes, including glucose levels, insulin sensitivity and oDI, were examined.

Consistent with the positive association of birthweight and newborn SSF with childhood insulin sensitivity, the present study demonstrated inverse associations of both birthweight and SSF with childhood glucose levels during an OGTT, associations that were strengthened by adjusting for maternal PG during pregnancy. The results of previous studies examining association of size at birth with childhood PG have been inconsistent. An inverse association and no association between ponderal index and fasting and post-load glucose levels have been demonstrated; however, some of the earlier studies focused primarily on small newborns [32-35]. Our results suggest that in a large, multi-ethnic, population-based cohort inverse associations between size at birth and post-load glucose values are present.

Our findings are somewhat counterintuitive. Greater newborn birthweight is typically associated with childhood obesity $[36,37]$, while greater adiposity in children is associated with insulin resistance [38]. This suggests that larger newborns would have higher glucose levels and greater 
Fig. 1 Association of birthweight, newborn SSF and cord C-peptide with childhood insulin sensitivity and oDI. The $\beta$ values (95\% CIs) for newborn birthweight (a), SSF (b) and cord C-peptide (c) higher by $1 \mathrm{SD}$ with the Matsuda index are shown, as are associations of birthweight (d), SSF (e) and cord C-peptide (f) higher by $1 \mathrm{SD}$ with oDI. Results are presented for each outcome for model 1 (M01, red) and model 2 (M02, blue)
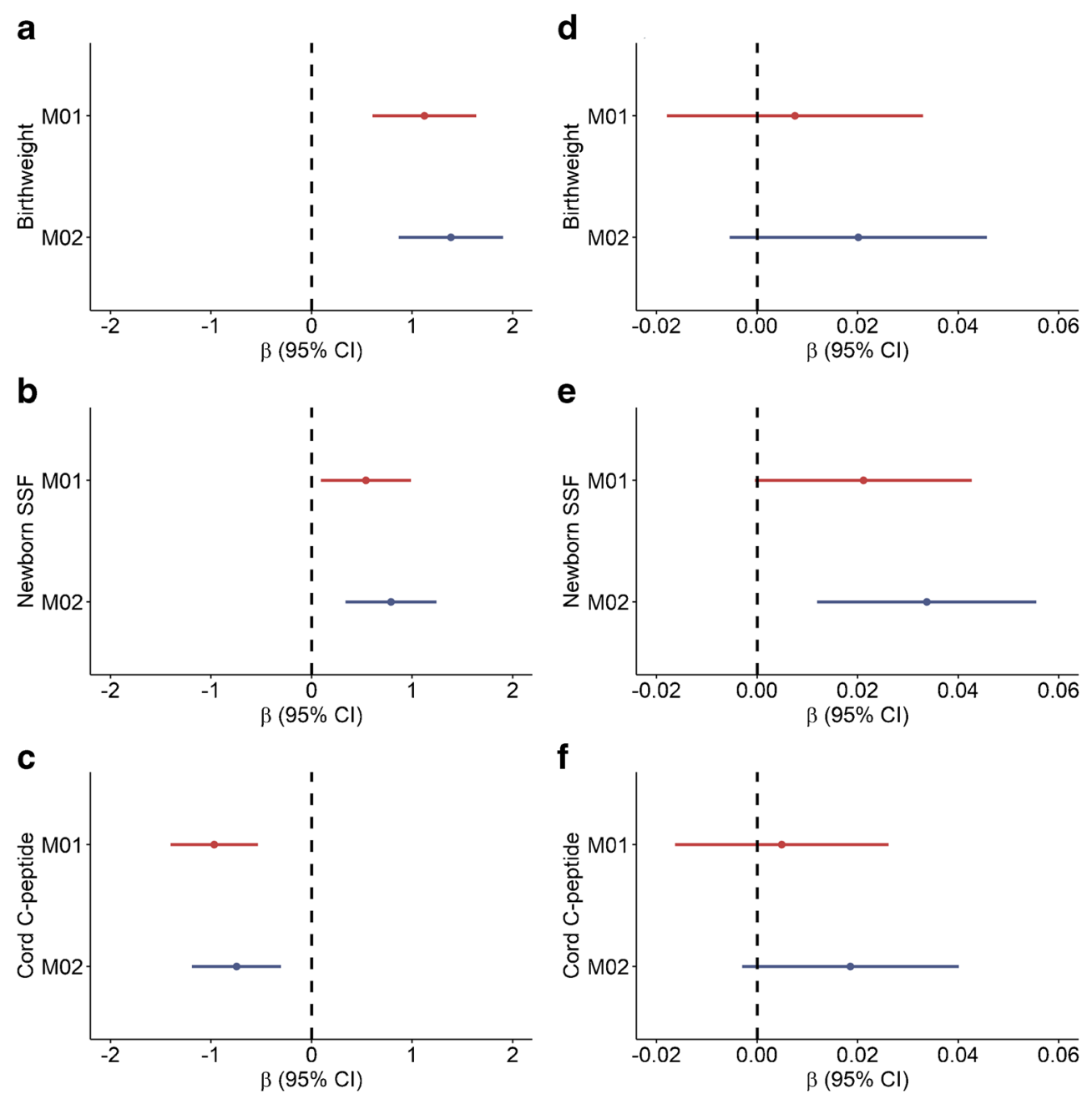

insulin resistance in childhood [39], which stands in contrast to the positive association of size at birth with childhood insulin sensitivity and inverse associations with glucose levels found in the present study. One potential explanation for our results is that newborns with higher birthweight may have more lean body mass during childhood, which would be associated with greater insulin sensitivity $[10,11,40]$. A positive relationship between birthweight and lean or fat-free mass during childhood has been demonstrated $[10,11]$.

The above explanation does not account for the observed association of newborn SSF, which reflects adiposity, with lower glucose levels and greater insulin sensitivity in childhood. Genetic variation determines, in part, birthweight and newborn adiposity [41-43]. One possibility is that genetic variation resulting in larger size at birth (including both lean body and fat mass) is associated with better metabolic health in childhood. This would be consistent with the observation that some genetic variants associated with lower birthweight are also associated with greater risk of type 2 diabetes, while genetic variants associated with a higher disposition index are associated with greater birthweight $[41,43]$. A second possible explanation, based upon the strengthening of the observed associations after adjustment for maternal glucose levels during pregnancy, is that additional fetal fat accretion that occurs secondary to maternal hyperglycaemia is associated with poorer metabolic health in childhood. This possibility is consistent with the observed associations of higher maternal glucose during pregnancy with greater newborn birthweight and adiposity as well as the association of higher maternal glucose levels during pregnancy with greater childhood adiposity and PG seen in the HAPO Study and HAPO FUS [18-21]. Future studies will be needed to examine these different possibilities.

In contrast to the findings with birthweight and newborn SSF, the only association observed for cord C-peptide was an inverse association with childhood insulin sensitivity (i.e., the higher the cord $\mathrm{C}$-peptide the greater the insulin resistance during childhood). One possible explanation for this inverse association is that it reflects an underlying genetic predisposition to insulin resistance that is first manifest as a high cord $\mathrm{C}$ peptide level. 

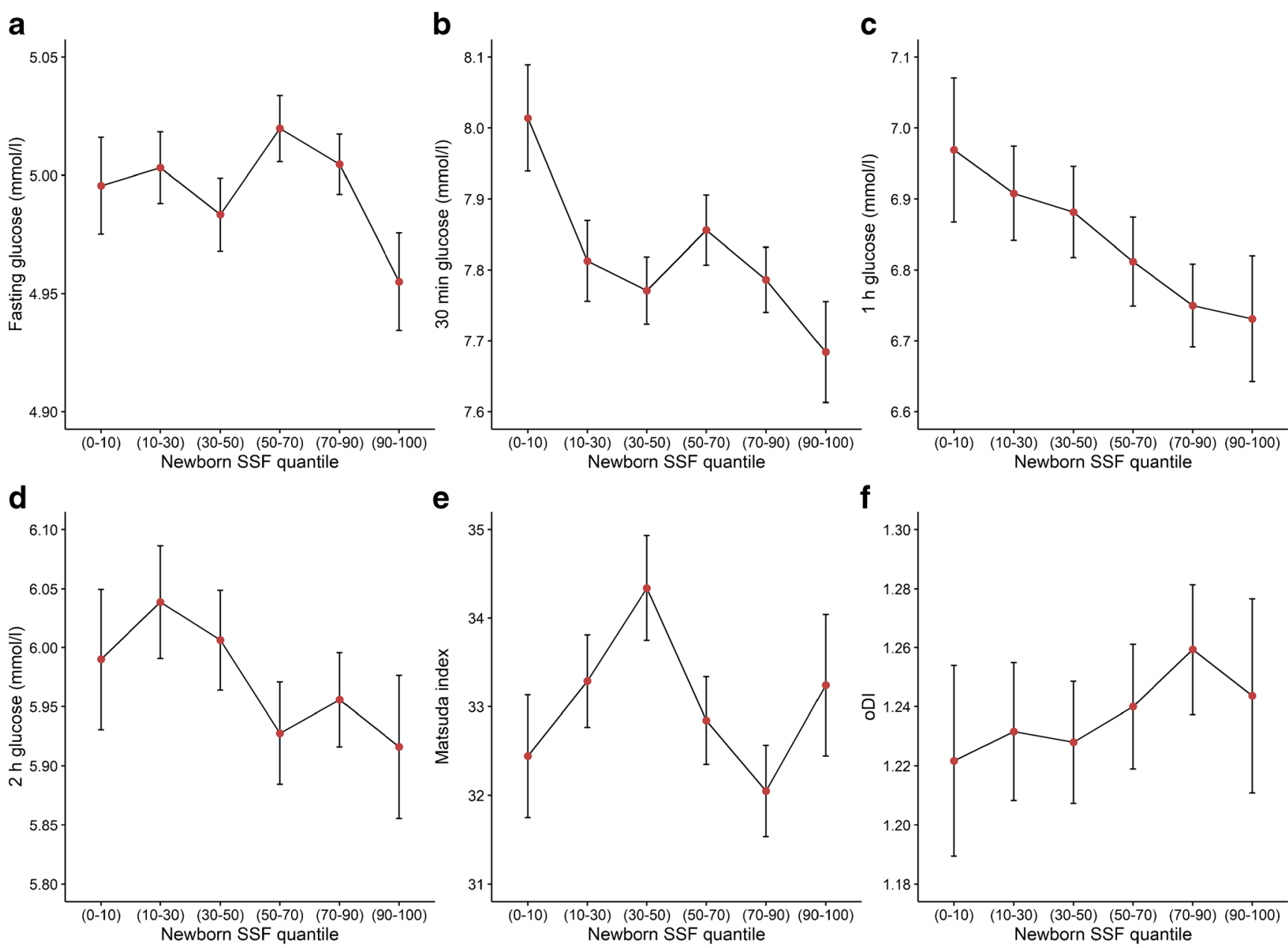

Fig. 2 Measures of glucose metabolism across SSF thickness quantiles. Quantiles of SSF ranging from $<10$ th percentile to $>90$ th percentile were determined. The mean \pm SEM of fasting (a), $30 \mathrm{~min}(\mathbf{b}), 1 \mathrm{~h}(\mathbf{c})$ and $2 \mathrm{~h} \mathrm{(d)}$ glucose as well as the Matsuda index (e) and oDI (f) across the quantiles of SSF were determined

to previous findings, higher birthweight and newborn adiposity are associated with greater childhood insulin sensitivity and lower glucose levels following a glucose load. Thus, greater size at birth per se may not necessarily be associated with adverse metabolic outcomes during early childhood. Future research that considers maternal metabolic factors during pregnancy will be required to replicate this finding and better define the complex associations among maternal PG during pregnancy, newborn adiposity and birthweight, and childhood metabolic outcomes.

Supplementary Information The online version contains peer-reviewed but unedited supplementary material available at https://doi.org/10.1007/ s00125-020-05331-0.

Acknowledgements Some of the data were presented as an abstract at the 101st Annual Meeting of the Endocrine Society in 2019. The HAPO Follow-Up Study investigators are grateful to all mothers and children who participated in HAPO and the HAPO Follow-Up Study.

Data availability Data and codes used for analyses will be made available by the authors upon request. 
Funding The HAPO Follow-Up Study is funded by grant 1U01DK 094830 from the National Institute of Diabetes, Digestive, and Kidney Diseases and by the Eunice Kennedy Shriver National Institute of Child Health and Human Development. The HAPO Study was funded by the Eunice Kennedy Shriver National Institute of Child Health and Human Development (R01-HD-34242 and R01-HD-34243). HAPO Follow-Up Study data were collected and managed using REDCap electronic data capture tools hosted at Northwestern University Feinberg School of Medicine (FSM). REDCap is supported at FSM by the Northwestern University Clinical and Translational Science (NUCATS) Institute. Research reported in this publication was supported, in part, by the National Institutes of Health's National Center for Advancing Translational Sciences, grants UL1TR001422 and TL1TR001423. The content is solely the responsibility of the authors and does not necessarily represent the official views of the National Institutes of Health.

Authors' relationships and activities The authors declare that there are no relationships or activities that might bias, or be perceived to bias, their work.

Contribution statement JLJ and PMC acquired data, interpreted the data and participated in writing the manuscript. MEB, BEM, LPL and WLL conceived and designed the study, researched the literature, interpreted the data and wrote the manuscript. ARD designed the study, acquired data, interpreted the data and wrote the manuscript. AK analysed the data and participated in writing the manuscript. DMS researched the literature, designed the study, acquired data, analysed the data, interpreted the data and wrote the manuscript. All authors reviewed the final version of the manuscript. WLL and DMS are the guarantors of this work and, as such, had full access to all of the data in the study and take responsibility for the integrity of the data and the accuracy of the data analysis.

\section{Appendix}

HAPO Follow-up Study Cooperative Research Group, field centre principal investigators and study staff

Bangkok C Deerochanawong, $\mathrm{T}$ Tanaphonpoonsuk (Rajavithi Hospital), S Binratkaew U Chotigeat, W Manyam, (Queen Sirikit National Institute of Child Health)

Barbados M Forde, A Greenidge, K Neblett, PM Lashley, D Walcott (Queen Elizabeth Hospital/School of Clinical Medicine and Research, University of the West Indies, Barbados)

Belfast K Corry, L Francis, J Irwin, A Langan, DR McCance, M Mousavi, (Belfast Health and Social Care Trust), IS Young (Queen's University Belfast)

Bellflower J Gutierrez, J Jimenez, JM Lawrence, DA Sacks, HS Takhar, E Tanton (Kaiser Permanente Southern California)

Chicago WJ Brickman, J Howard, JL Josefson, L Miller, R Steuer (Ann and Robert H Lurie Children's Hospital/Northwestern University Feinberg School of Medicine)

Cleveland J Bjaloncik, PM Catalano, A Davis, K Koontz, L Presley, S Smith, A Tyhulski (MetroHealth Medical Center/Case Western Reserve University)

Hong Kong A Li, RC Ma, R Ozaki, WH Tam, M Wong, C Yuen (The Chinese University of Hong Kong/Prince of Wales Hospital)

Manchester PE Clayton, A Khan, A Vyas (Royal Manchester Children's Hospital, Manchester University Hospitals NHS Foundation Trust, Manchester Academic Healthy Sciences Centre/School of Medical Sciences, Faculty of Biology, Medicine \& Health, University of Manchester), M Maresh (St. Mary's Hospital, Central Manchester University Hospitals NHS Foundation Trust, Manchester Academic Health Sciences Centre)

Petah-Tiqva H Benzaquen, N Glickman, A Hamou, O Hermon, O Horesh, Y Keren, S Shalitin (Schneider Children's Medical Center of Israel), Y Lebenthal (Jesse Z and Sara Lea Shafer Institute for
Endocrinology and Diabetes, National Center for Childhood Diabetes, Schneider Children's Medical Center of Israel, Sackler Faculty of Medicine, Tel Aviv University)

Toronto K Cordeiro, J Hamilton, HY Nguyen, S Steele (The Hospital for Sick Children, University of Toronto)

Coordinating Centers: F Chen, AR Dyer, W Huang, A Kuang, M Jimenez, LP Lowe, WL Lowe, Jr, BE Metzger, M Nodzenski, A Reisetter, D Scholtens, P Yim (Northwestern University Feinberg School of Medicine)

Consultants: D Dunger, A Thomas

NIDDK: M Horlick, B Linder, A Unalp-Arida

NICHD: G Grave

\section{References}

1. Dabelea D, Mayer-Davis EJ, Saydah S et al (2014) Prevalence of type 1 and type 2 diabetes among children and adolescents from 2001 to 2009. JAMA 311:1778-1786. https://doi.org/10.1001/ jama.2014.3201

2. Imperatore G, Boyle JP, Thompson TJ et al (2012) Projections of type 1 and type 2 diabetes burden in the U.S. population aged $<20$ years through 2050: dynamic modeling of incidence, mortality, and population growth. Diabetes Care 35:2515-2520. https://doi.org/ $10.2337 / \mathrm{dc} 12-0669$

3. The RISE Consortium (2018) Metabolic Contrasts Between Youth and Adults With Impaired Glucose Tolerance or Recently Diagnosed Type 2 Diabetes: I. Observations Using the Hyperglycemic Clamp. Diabetes Care 41:1696-1706. https://doi. org/10.2337/dc18-0244

4. TODAY Study Group, Zeitler P, Hirst K et al (2012) A clinical trial to maintain glycemic control in youth with type 2 diabetes. N Engl J Med 366:2247-2256. https://doi.org/10.1056/NEJMoa1 109333

5. TODAY Study Group (2013) Effects of metformin, metformin plus rosiglitazone, and metformin plus lifestyle on insulin sensitivity and beta-cell function in TODAY. Diabetes Care 36:1749-1757. https://doi.org/10.2337/dc12-2393

6. Fagot-Campagna A, Pettitt DJ, Engelgau MM et al (2000) Type 2 diabetes among North American children and adolescents: an epidemiologic review and a public health perspective. J Pediatr 136:664-672. https://doi.org/10.1067/mpd.2000.105141

7. Bush NC, Chandler-Laney PC, Rouse DJ, Granger WM, Oster RA, Gower BA (2011) Higher maternal gestational glucose concentration is associated with lower offspring insulin sensitivity and altered beta-cell function. J Clin Endocrinol Metab 96:E803-E809. https:// doi.org/10.1210/jc.2010-2902

8. Metzger BE, Lowe LP, Dyer AR et al (2008) Hyperglycemia and adverse pregnancy outcomes. N Engl J Med 358:1991-2002. https://doi.org/10.1056/NEJMoa0707943

9. Lawlor DA, Riddoch CJ, Page AS et al (2005) The association of birthweight and contemporary size with insulin resistance among children from Estonia and Denmark: findings from the European Youth Heart Study. Diabet Med 22:921-930. https://doi.org/10. 1111/j.1464-5491.2005.01551.x

10. Murphy MJ, Metcalf BS, Jeffery AN, Voss LD, Wilkin TJ (2006) Does lean rather than fat mass provide the link between birth weight, BMI, and metabolic risk? EarlyBird 23. Pediatr Diabetes 7:211-214. https://doi.org/10.1111/j.1399-5448.2006.00180.x

11. Singhal A, Wells J, Cole TJ, Fewtrell M, Lucas A (2003) Programming of lean body mass: a link between birth weight, obesity, and cardiovascular disease? Am J Clin Nutr 77:726-730. https://doi.org/10.1093/ajcn/77.3.726 
12. Van Hulst A, Paradis G, Benedetti A, Barnett TA, Henderson M (2018) Pathways linking birth weight and insulin sensitivity in early adolescence: a double mediation analysis. J Clin Endocrinol Metab 103:4524-4532. https://doi.org/10.1210/jc.2018-00525

13. Whitrow MJ, Davies MJ, Giles LC et al (2013) Effects of birth size, post-natal growth and current size on insulin resistance in 9-yearold children: a prospective cohort study. Eur J Pediatr 172:12071214. https://doi.org/10.1007/s00431-013-2017-4

14. Johnson L, Llewellyn CH, van Jaarsveld CH, Cole TJ, Wardle J (2011) Genetic and environmental influences on infant growth: prospective analysis of the Gemini twin birth cohort. PLoS One 6: e19918. https://doi.org/10.1371/journal.pone.0019918

15. Norris SA, Osmond C, Gigante D et al (2012) Size at birth, weight gain in infancy and childhood, and adult diabetes risk in five low- or middle-income country birth cohorts. Diabetes Care 35:72-79. https://doi.org/10.2337/dc11-0456

16. Whincup PH, Kaye SJ, Owen CG et al (2008) Birth weight and risk of type 2 diabetes: a systematic review. JAMA 300:2886-2897. https://doi.org/10.1001/jama.2008.886

17. Dyck RF, Klomp H, Tan L (2001) From "thrifty genotype" to "hefty fetal phenotype": the relationship between high birthweight and diabetes in Saskatchewan Registered Indians. Can J Public Health 92:340-344. https://doi.org/10.1007/BF03404975

18. HAPO Study Cooperative Research Group (2009) Hyperglycemia and Adverse Pregnancy Outcome (HAPO) Study: associations with neonatal anthropometrics. Diabetes 58:453-459. https://doi.org/10. 2337/db08-1112

19. Lowe WL Jr, Scholtens DM, Kuang A et al (2019) Hyperglycemia and Adverse Pregnancy Outcome Follow-up Study (HAPO FUS): maternal gestational diabetes mellitus and childhood glucose metabolism. Diabetes Care 42:372-380. https://doi.org/10.2337/ dc18-1646

20. Scholtens DM, Kuang A, Lowe LP et al (2019) Hyperglycemia and Adverse Pregnancy Outcome Follow-up Study (HAPO FUS): maternal glycemia and childhood glucose metabolism. Diabetes Care 42:381-392. https://doi.org/10.2337/dc18-2021

21. Lowe WL Jr, Scholtens DM, Lowe LP et al (2018) Association of gestational diabetes with maternal disorders of glucose metabolism and childhood adiposity. JAMA 320:1005-1016. https://doi.org/ 10.1001/jama.2018.11628

22. Matsuda M, DeFronzo RA (1999) Insulin sensitivity indices obtained from oral glucose tolerance testing: comparison with the euglycemic insulin clamp. Diabetes Care 22:1462-1470. https:// doi.org/10.2337/diacare.22.9.1462

23. Phillips DI, Clark PM, Hales CN, Osmond C (1994) Understanding oral glucose tolerance: comparison of glucose or insulin measurements during the oral glucose tolerance test with specific measurements of insulin resistance and insulin secretion. Diabet Med 11: 286-292. https://doi.org/10.1111/j.1464-5491.1994.tb00273.x

24. Weiss R, Cali AM, Dziura J, Burgert TS, Tamborlane WV, Caprio S (2007) Degree of obesity and glucose allostasis are major effectors of glucose tolerance dynamics in obese youth. Diabetes Care 30:1845-1850. https://doi.org/10.2337/dc07-0325

25. Harrell Jr. FE (2018) "rms: Regression Modeling Strategies." R package version 5.1-2. Available from https://cran.r-project.org/ src/contrib/Archive/rms/rms_5.1-2.tar.gz. Accessed 30/03/2018

26. Catalano PM, McIntyre HD, Cruickshank JK et al (2012) The hyperglycemia and adverse pregnancy outcome study: associations of GDM and obesity with pregnancy outcomes. Diabetes Care 35: 780-786. https://doi.org/10.2337/dc11-1790

27. Van Buuren S, Groothuis-Oudshoorn K (2011) Multivariate imputation by chained equations in R. J Stat Softw 45:1-67. https://doi. org/10.18637/jss.v045.103

28. White IR, Daniel R, Royston P (2010) Avoiding bias due to perfect prediction in multiple imputation of incomplete categorical variables. Comput Stat Data Anal 54:2267-2275. https://doi.org/ 10.1016/j.csda.2010.04.005

29. R Core Team (2016) R: a language and environment for statistical computing. R Foundation for Statistical Computing, Vienna

30. Johansson S, Iliadou A, Bergvall $\mathrm{N}$ et al (2008) The association between low birth weight and type 2 diabetes: contribution of genetic factors. Epidemiology 19:659-665. https://doi.org/10. 1097/ede.0b013e31818131b9

31. Harder T, Rodekamp E, Schellong K, Dudenhausen JW, Plagemann A (2007) Birth weight and subsequent risk of type 2 diabetes: a meta-analysis. Am J Epidemiol 165:849-857. https:// doi.org/10.1093/aje/kwk071

32. Whincup PH, Cook DG, Adshead F et al (1997) Childhood size is more strongly related than size at birth to glucose and insulin levels in 10-11-year-old children. Diabetologia 40:319-326. https://doi. org/10.1007/s001250050681

33. Forrester TE, Wilks RJ, Bennett FI et al (1996) Fetal growth and cardiovascular risk factors in Jamaican schoolchildren. BMJ 312 156-160. https://doi.org/10.1136/bmj.312.7024.156

34. Yajnik CS, Fall CH, Vaidya U et al (1995) Fetal growth and glucose and insulin metabolism in four-year-old Indian children. Diabet Med 12:330-336. https://doi.org/10.1111/j.1464-5491. 1995.tb00487.x

35. Law CM, Gordon GS, Shiell AW, Barker DJ, Hales CN (1995) Thinness at birth and glucose tolerance in seven-year-old children. Diabet Med 12:24-29. https://doi.org/10.1111/j.1464-5491.1995. tb02057.x

36. Sacco MR, de Castro NP, Euclydes VL, Souza JM, Rondo PH (2013) Birth weight, rapid weight gain in infancy and markers of overweight and obesity in childhood. Eur J Clin Nutr 67:11471153. https://doi.org/10.1038/ejen.2013.183

37. Oken E, Gillman MW (2003) Fetal origins of obesity. Obes Res 11: 496-506. https://doi.org/10.1038/oby.2003.69

38. Kim JY, Bacha F, Tfayli H, Michaliszyn SF, Yousuf S, Arslanian S (2019) Adipose Tissue Insulin Resistance in Youth on the Spectrum From Normal Weight to Obese and From Normal Glucose Tolerance to Impaired Glucose Tolerance to Type 2 Diabetes. Diabetes Care 42:265-272. https://doi.org/10.2337/dc18-1178

39. Catalano PM, Farrell K, Thomas A et al (2009) Perinatal risk factors for childhood obesity and metabolic dysregulation. Am J Clin Nutr 90:1303-1313. https://doi.org/10.3945/ajcn.2008.27416

40. Fall CH (2011) Evidence for the intra-uterine programming of adiposity in later life. Ann Hum Biol 38:410-428. https://doi.org/ 10.3109/03014460.2011.592513

41. Horikoshi M, Yaghootkar H, Mook-Kanamori DO et al (2013) New loci associated with birth weight identify genetic links between intrauterine growth and adult height and metabolism. Nat Genet 45:76-82. https://doi.org/10.1038/ng.2477

42. Urbanek M, Hayes MG, Armstrong LL et al (2013) The chromosome $3 \mathrm{q} 25$ genomic region is associated with measures of adiposity in newborns in a multi-ethnic genome-wide association study. Hum Mol Genet 22:3583-3596. https://doi.org/10.1093/hmg/ddt168

43. Warrington NM, Beaumont RN, Horikoshi M et al (2019) Maternal and fetal genetic effects on birth weight and their relevance to cardio-metabolic risk factors. Nat Genet 51:804-814. https://doi. org/10.1038/s41588-019-0403-1

44. Eriksson JG, Forsen T, Tuomilehto J, Osmond C, Barker DJ (2003) Early adiposity rebound in childhood and risk of Type 2 diabetes in adult life. Diabetologia 46:190-194. https://doi.org/10.1007/ s00125-002-1012-5

Publisher's note Springer Nature remains neutral with regard to jurisdictional claims in published maps and institutional affiliations. 